\title{
Gut Microbiota: Novel Therapeutic Target of Ginsenosides for the Treatment of Obesity and Its Complications
}

\author{
Tongxi Zhuang ${ }^{1,2+}$, Wei $\mathrm{Li}^{1,3}$, Li Yang ${ }^{1,3}$, Zhengtao Wang ${ }^{1,3}$, Lili Ding ${ }^{1,3 *}$ and Mingmei Zhou ${ }^{2 *}$ \\ ${ }^{1}$ Shanghai Key Laboratory of Complex Prescriptions and MOE Key Laboratory for Standardization of Chinese Medicines, Institute \\ of Chinese Materia Medica, Shanghai University of Traditional Chinese Medicine, Shanghai, China, ${ }^{2}$ Center for Chinese Medicine \\ Therapy and Systems Biology, Institute for Interdisciplinary Medicine Sciences, Shanghai University of Traditional Chinese \\ Medicine, Shanghai, China, ${ }^{3}$ Shanghai R\&D Center for Standardization of Traditional Chinese Medicines, Shanghai, China
}

OPEN ACCESS

Edited by:

Ching-feng Cheng,

Taipei Tzu Chi Hospital, Taiwan

Reviewed by:

Min Wu,

China Academy of Chinese Medical

Sciences, China

Sofia Viana,

University of Coimbra, Portugal Yu-Cheng Lin,

Far Eastern Memorial Hospital (FEMH), Taiwan

*Correspondence:

Lili Ding

nail8219@126.com

Mingmei Zhou

zhoumm368@163.com

${ }^{\dagger}$ These authors share first authorship

Specialty section:

This article was submitted to

Ethnopharmacology,

a section of the journal

Frontiers in Pharmacology

Received: 26 June 2021 Accepted: 16 August 2021 Published: 27 August 2021

Citation:

Zhuang T, Li W, Yang L, Wang Z,

Ding $L$ and Zhou M (2021) Gut Microbiota: Novel Therapeutic Target of Ginsenosides for the Treatment of

Obesity and Its Complications.

Front. Pharmacol. 12:731288.

doi: 10.3389/fphar.2021.731288
Obesity, generally characterized by excessive lipid accumulation, is a metabolic threat worldwide due to its rapid growth in global prevalence. Ginsenosides are crucial components derived from natural plants that can confer metabolic benefits for obese patients. Considering the low bioavailability and degradable properties of ginsenosides in vivo, it should be admitted that the mechanism of ginsenosides on anti-obesity contribution is still obscure. Recently, studies have indicated that ginsenoside intervention has beneficial metabolic effects on obesity and its complications because it allows for the correction of gut microbiota dysbiosis and regulates the secretion of related endogenous metabolites. In this review, we summarize the role of gut microbiota in the pathogenetic process of obesity, and explore the mechanism of ginsenosides for ameliorating obesity, which can modulate the composition of gut microbiota by improving the metabolism of intestinal endogenous substances and alleviating the level of inflammation. Ginsenosides are expected to become a promising anti-obesity medical intervention in the foreseeable clinical settings.

Keywords: obesity, gut microbiota, ginsenosides, mechanism, target

\section{INTRODUCTION}

Obesity, a complicated chronic metabolic disorder (An et al., 2020), causes visual changes in humans, alongside the excessive accumulation of adipose tissue (Hammarstedt et al., 2018; Ruiz-Ojeda et al., 2019). Long-term imbalance between calorie intake and metabolic consumption is the main cause of obesity, which can also lead to increases in systemic inflammation levels and excessive oxidative stress (Karam et al., 2017; Brooks-Worrell and Palmer, 2019). A strong correlation exists between obesity and complications like diabetes, nonalcoholic fatty liver disease (NAFLD) (Xiao et al., 2017) and increased mortality (Jung and Choi, 2014; Leoni et al., 2018; Tate et al., 2020). The menace of obesity and its complications within our already overburdened healthcare systems is staggering.

Ginsenosides, commonly attributed to triterpenoid saponins in botany, mainly exist in the plants of ginseng, Korean ginseng and Panax notoginseng. (Kim D. et al., 2018; Zhang et al., 2021). Ginsenosides are generally divided into three forms (Figure 1): oleanolic acid pentacyclic triterpenoid saponin Ro, which is exceedingly low in concentration and thus rarely detected; as well as panaxadiol saponins (such as $\mathrm{Rb} 1, \mathrm{Rb} 2, \mathrm{Rc}, \mathrm{Rd}, \mathrm{F} 2, \mathrm{Rg} 3, \mathrm{Rh} 2$, etc.) and panaxatriol 


\section{Protopanaxadiol type(PPD)}

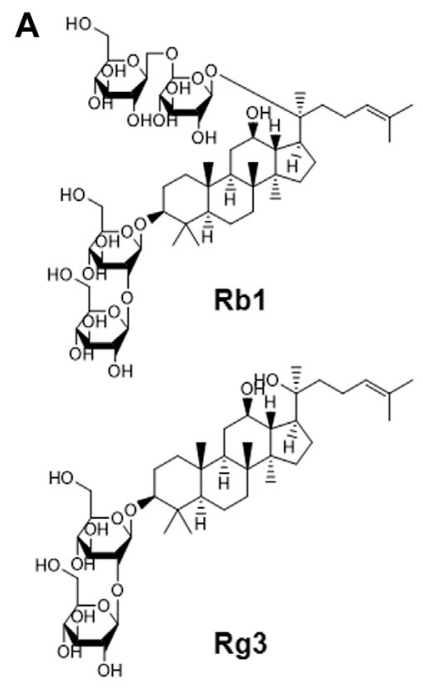

Protopanaxatriol type (PPT)

B
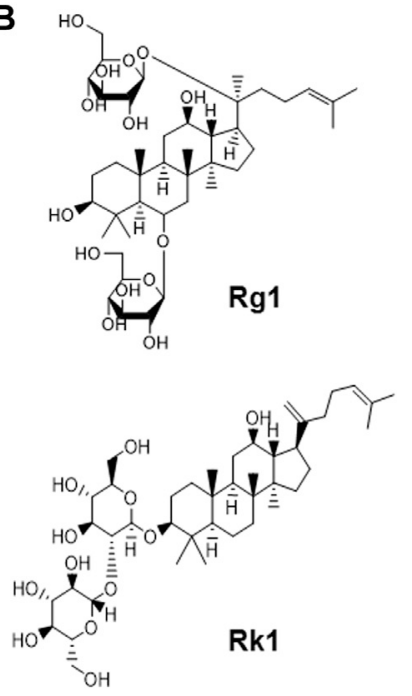

\section{Oleanolic acids type (OA)}

C

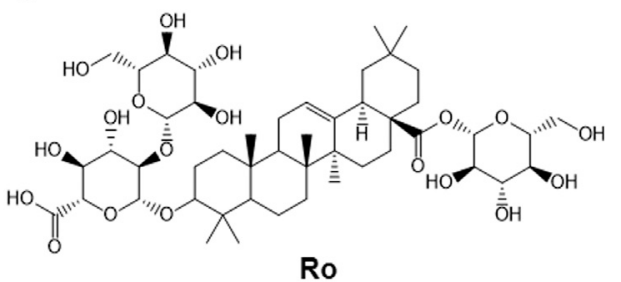

D

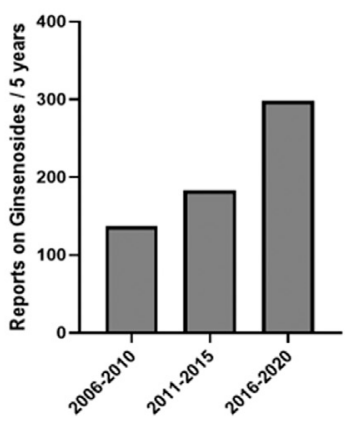

FIGURE 1 | Chemical structures of representative primary ginsenosides from ginseng for improving obesity and its complications targeting at gut microbiota. (A). Protopanaxadiol type(PPD), (B). Protopanaxatriol type (PPT), (C). Oleanolic acids type (OA), (D). Literature deposited in the PubMed database from 2006 to 2020 using ginsenosides, obesity and complications as subject terms.

saponins (such as Re, Rg1, Rg2, Rh1, etc.), both of which are dammarane-type tetracyclic triterpenoid saponins (Sun et al., 2006; Choi, 2008). Modern pharmacological studies have suggested that ginsenosides have good anti-tumor, antiinflammatory, antioxidant and anti-apoptosis properties (Shibata, 2001; Kim et al., 2017; Liu et al., 2019; Yao et al., 2019). In addition, recent studies have shown that multiple ginsenosides improve obesity by regulating different targets. For instance, ginsenoside $\mathrm{Mcl}$ has been shown to ameliorate glucose intolerance and insulin resistance by suppressing c-Jun N-terminal kinase (JNK) phosphorylation (Roh et al., 2020). Ginsenoside Rb1 extracted from crude saponins of Korea red ginseng has also become a promising product for the treatment of obesity and related metabolic disorders by modulating peripheral and central appetite-regulating signals (Park et al., 2019). Ginsenoside Rg3 promoted the browning of white fat in obese mice, as well as significantly ameliorating body weight and blood lipid profiles (Mu et al., 2021). Meanwhile, it is worth noting that, in two clinical investigations, Korean red ginseng failed to improve the insulin sensitivity of non-diabetic healthy overweight and obese adults, and oral ginsenoside Re therapy was unable to upregulate insulin sensitivity in overweight and obese subjects with impaired glucose tolerance or newly diagnosed diabetes, which indicated that certain ginsenosides or ginseng processing drug may have no significant effect on the recovery of insulin sensitivity in obese patients (Reeds et al., 2011; Cho et al., 2013). In summary, although the efficacy of Ginsenoside in improving insulin sensitivity is questionable, ginsenosides show strong potential for regulating glucose and lipid metabolism, and thus conferring metabolic benefits on obesity.
With increased understanding of gut microbiota and metabolomics in recent years, we now know that onset of metabolic diseases is closely related to changes in gut microbiota composition (Devaraj et al., 2013; Mei et al., 2016; Kootte et al., 2017). Gut microbiota is commonly considered to be indispensable normal microorganisms that reside in the intestine. Different forms of bacterial subtypes restrict and depend on one another in order to form a qualitative and quantitative ecological balance. Thus, gut microbiota constitutes a balanced system, and participate in many physiological and pathological processes (Ley et al., 2006; Saad et al., 2016; Pickard et al., 2017). In the past few decades, studies have shown that gut microbiota dysfunction might induce obesity and provoke serious stress responses (Tsai and Coyle, 2009). Ley first identified the correlation between obesity and gut microbiota, finding that Bacteroides decreased in obese mice, but that increases in Firmicutes occurred in the same model (Ley et al., 2005). Subsequent research on Enterobacter cloacae in the gut microbiota showed that its overgrowth in the human intestinal tract directly led to obesity (Keskitalo et al., 2018), suggesting a causal link between gut microbiota and obesity. Thus, gut microbiota may be a noteworthy target for the treatment of obesity.

An interesting phenomenon has been discovered is that the obesity-improving effects of ginsenosides, which work by acting on the gut microbiota, may be bidirectional. On one hand, some reports have shown that ginsenosides regulate gut microbiota disorders by restoring normal structural composition and thereby improving the obesity phenotype. Gut microbiota is a novel therapeutic target of ginsenosides, and it is expected that ginsenosides will be promoted as a clinical drug for obesity and its complications. On the other hand, the content of 


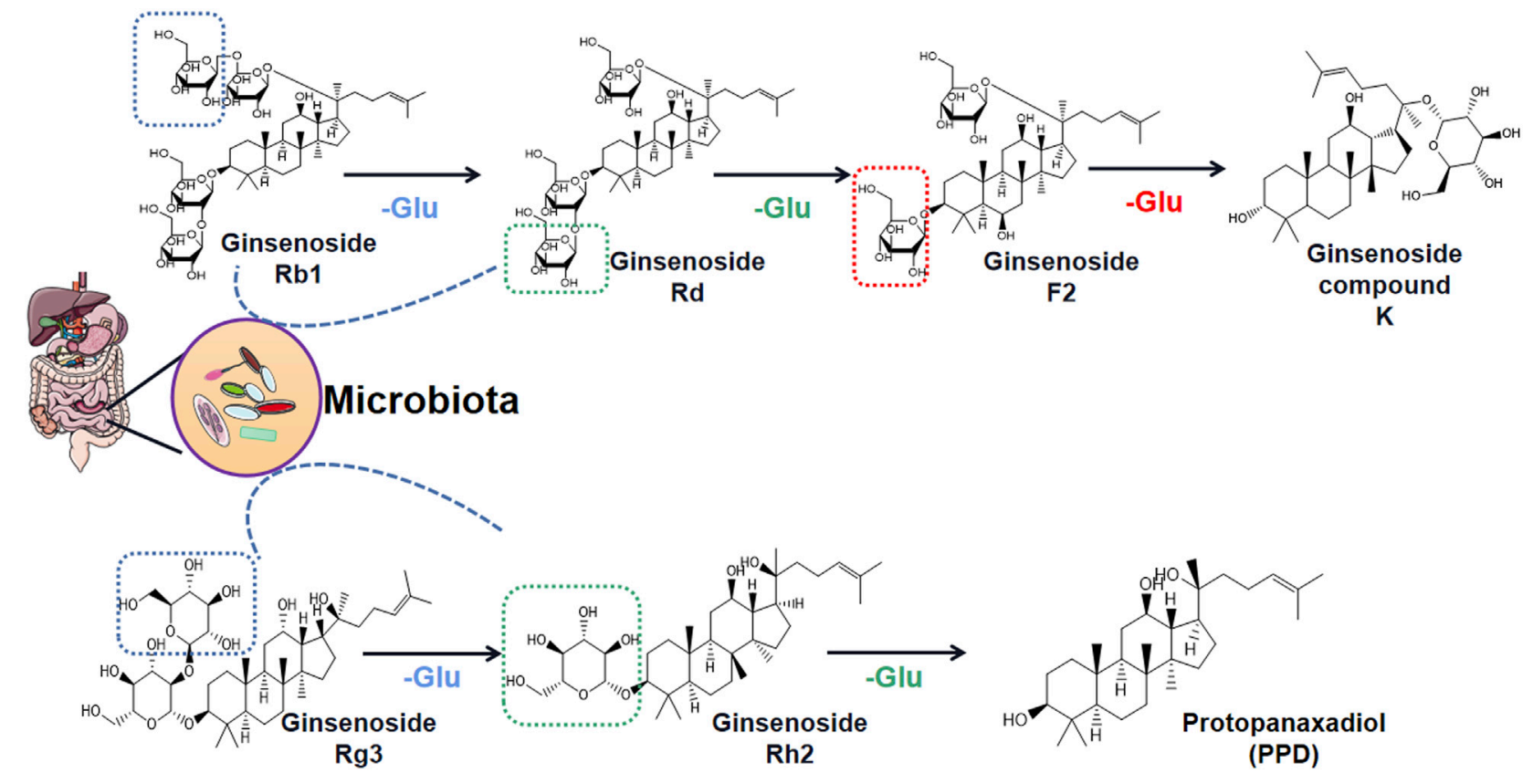

FIGURE 2 | Deglycosylation process of ginsenosides Rb1 and Rg3 through gut microbiota regulation.

ginsenosides in ginseng species is extremely rare, and most ginsenoside monomers have inherent clinical disadvantages, such as low water solubility, fast elimination rate, and low bioavailability (Pan et al., 2018; Yang et al., 2020). So how do they exert their weight loss effects? In recent years, many studies have revealed that ginsenosides are biodegraded by gut microbiota and are metabolized into monoglycosides and aglycons through deglycosylation reactions (Figure 2) (Akao et al., 1998; Li R. et al., 2018). Thus, both ginsenosides and gut microbiota may have complementary, symbiotic effects on obesity rather than their interaction being a case of simple target action.

In this review, we clarify the mechanisms of gut microbiota for addressing the pathophysiology of obesity. In addition, we describe the significance of gut microbiota as a target for ginsenosides' anti-obesity effects, in order to enrich future theoretical research on the mechanism of ginsenosides in the context of their clinical application, and also to provide new insight around the power of natural products for ameliorating metabolic diseases.

\section{THE ROLE OF GUT MICROBIOTA DYSBIOSIS IN THE PATHOPHYSIOLOGICAL MECHANISM OF OBESITY}

Diet is an indispensable external factor that affects gut microbiota composition (David et al., 2014; Sonnenburg and Bäckhed, 2016). The primary initiators of obesity are decreased exercise and increased intake of high-energy food. In addition, various gene polymorphisms have significant effects on the pathogenesis of obesity (Huang et al., 2018; Kim HJ. et al., 2018). Specific proteins and hormone factors play a role in regulating metabolism and body weight. The composition of human intestinal microflora is transformed when diets are changed (Scott et al., 2013; Gentile and Weir, 2018). Consequently, the alteration of gut microbiota will impact fatty acid metabolism, affecting multiple inflammatory factors signaling pathways and even the original environment of the intestinal tract. After this, deposition of massive adipose tissue will emerge, eventually damaging the host's homeostasis (Figure 3) (Kobyliak et al., 2016; Hersoug et al., 2018; Torres et al., 2019).

\section{Gut Microbiota Dysbiosis Can Inhibit Short Chain Fatty Acids Oxidation}

Short chain fatty acids (SCFAs) are some of elementary substances involved in the synthesis of human adipose tissue. They also serve an important role in the obesity process by regulating triglycerides, adipocyte differentiation, and leptin expression (Tan et al., 2014; De la Cuesta-Zuluaga et al., 2019; Jiao et al., 2020). Once gut microbiota falls into a state of disorder, SCFAs are spontaneously influenced by varying proportions of Bacteroides and Pachytene, resulting in decreases in butyrate and crippled expression abilities of $\mathrm{G}$ protein-coupled receptor 41 (GPR41) and G protein-coupled receptor 43 (GPR43) (Ang and Ding, 2016; Lu et al., 2016). The oxidation process of SCFAs can be reversed, however, by inhibiting the phosphorylation of peroxisome proliferator-activated receptor $\gamma$ coactivator $1 a$ (PGC-1 $\alpha$ ), and inhibiting the expression of mitochondrial 


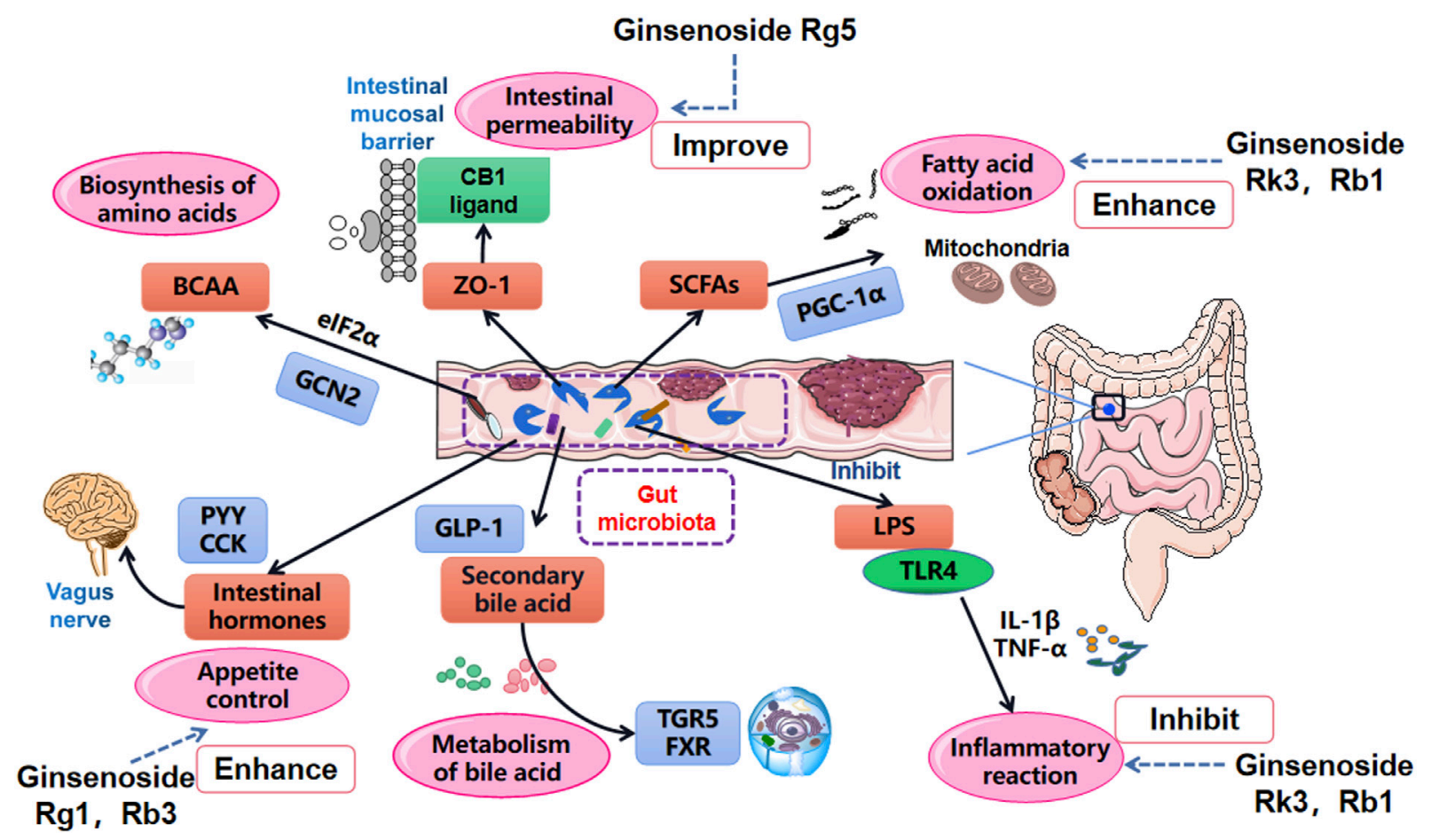

FIGURE 3 | Main influential pathways for obesity improvement via the role of gut microbiota manipulation. Gut microbiota affects the oxidation of SCFAs, the absorption of LPS and inflammation level, changes intestinal mucosal permeability by acting on tight junction protein ZO-1, manages the metabolism of bile acids by regulating TGR5 and FXR, influences the metabolism of BCAA and release of intestinal hormones.

uncoupling protein-1 (UCP-1) in brown adipose tissue (BAT) (Argentato et al., 2018; Wang et al., 2020). In addition, research has shown that gut flora partly inhibits intestinal fasting-induced adipocyte factor (FIAF) by promoting agonistic activity of the lipoprotein lipase in adipocytes and thus decreasing lipid deposition and storage (Bäckhed et al., 2004; Aronsson et al., 2010; Guo et al., 2017). Increased FIAF has also been shown to stimulate the production of PGC-1 $1 \alpha$, upregulate the expression of the fatty acid oxidation gene in mitochondria, and complete fatty acid oxidation (Alex et al., 2013; Xiao et al., 2019).

\section{Gut Microbiota Dysbiosis Can Increase the Absorption of Lipopolysaccharide and \\ Cause Inflammation}

LPS (lipopolysaccharide) is a common component of Gramnegative bacteria in the gut microbiota. Researchers have identified that LPS levels in rats fed with a high-fat diet were significantly higher than those in the control group, and that rats in the high-fat diet group existed in a sustained low-grade inflammation state (Bernard et al., 2019; Zhang H. et al., 2020). Cani named the host inflammatory response induced by low level LPS as "metabolic endotoxemia" (Cani et al., 2007). He believed that LPS was one of the major risk factors for metabolic disorders, and helped explain why there was a causal link between gut microbiota and obesity (Cani et al., 2008; Everard et al., 2013). Further study found that this LPS-mediated inflammation could be reversed. The obesity metabolic phenotype of mice was significantly improved when CD14 was eliminated, and when antibiotic interventions were used to reduce the inflammatory state caused by lipopolysaccharides (Carvalho et al., 2012; Boutens et al., 2018).

Increased LPS levels may be related to obesity. Certain diets can induce leptin resistance in the vagus nerve afferent of obese mice, and LPS is believed to play a role in this resistance (De Lartigue et al., 2011). Gut microbiota is involved in the inflammatory and obesity process by stimulating host appetite (Torres-Fuentes et al., 2017). LPS-induced obesity has also been causally linked to the binding of scavenger receptor type B (SR-BI) (Hersoug et al., 2016). These binding products enhanced LPS' actions (i.e., promoted the transport of lipoproteins on the endothelial barrier and the endocytosis of adipocytes), accelerated the transformation of macrophages from M2 type to M1 type in adipose tissue, and mediated inflammatory reactions (Xiong et al., 2018; O'Reilly et al., 2019). In addition, it has been shown that LPS contributes to the expression of receptor protein mRNA that is related to angiotensin receptors, and that this expression was positively correlated with specific bacteria in the gut (Oliveira et al., 2020).

\section{Gut Microbiota Dysbiosis Can Change Intestinal Mucosal Permeability}

Intestinal homeostasis is a dynamic balance formed by the interactions between the intestinal mucosal barrier and the gut 
microenvironment and its metabolites. The intestinal mucosal barrier prevents the invasion of pathogenic antigens and maintains intestinal health (Martens et al., 2018). Gut microbiota plays a crucial role in influencing tight junctions (TJ) and intestinal epithelial permeability to enhance the stability of intestinal epithelial cells.

Cumulative high-fat diets induce lesions in the composition of gut microbiota, and thus are also linked to intestinal epithelial damage. Gut microbiota disorders can activate intestinal epidermal growth factors, which directly act on protease activated receptors, cripple intestinal TJ structure, significantly down-regulate the expression of TJ protein zonula occludens 1 (ZO-1), eventually destroying the structure of intestinal barrier and increasing the amount of lipopolysaccharide entering the blood from the intestinal tract (Guo et al., 2013; Pontarollo et al., 2020). It has been found that gut microbiota affects the permeability of intestinal barrier partly via the actions of glucagon like peptide 2 (GLP-2) (Cani et al., 2009). When the proportion of Lactobacillus and Bifidobacterium in the intestinal tract is decreased, the production of intestinal GLP-2 is also reduced. GLP-2 has also been shown to weaken the tight connection between intestinal epithelial cells, increase the amount of lipopolysaccharides in the blood, and ultimately aggravate the inflammatory system (Watson and Duckworth, 2010). By employing prebiotics to maintain the quantity of Bifidobacteria in the host gut, gut barrier damages can be reversed: mice treated with prebiotics showed resistance to endotoxin level increases and obesity that would have been induced by high-fat diets (Krumbeck et al., 2018). Kuhn found that intraepithelial lymphocytes (IEL), a main IL-6 producer, are located at the epithelial barrier and maintain intestinal epithelial homeostasis (Regner et al., 2018). Bacteria in Bacteroides promote the presence of IEL in the colon and reduce the integrity of the intestinal epithelium by reducing the expression of blocked protein 1 and thinning the gel layer (Kuhn et al., 2018).

\section{Gut Microbiota Dysbiosis Can Change the Metabolism of Bile Acids}

Bile acids (BAs) are a crucial component of bile, and regulate lipid metabolism by controlling the activity of pancreatic lipase and lipoprotein esterase, and by improving the metabolism of fat hydrolysis. BAs are also responsible for lipid transport in the gut and fat absorption (Chávez-Talavera et al., 2017). On the whole, routine BA manipulation is inseparable from regulation of the gut microbiota. Former research has shown that mice treated with antibiotics showed increased intestinal bile acid reabsorption, and significantly lowered bile acid excretion in their feces (Behr et al., 2019). Farnesoid X receptor (FXR) and G protein coupled bile receptor 5 (TGR5) are classic bile acid receptors transformed by intestinal microorganisms, which are responsible for the balance of bile acid, lipid and glucose homeostasis in the host liver, intestine and peripheral tissues (Chiang, 2017; Jadhav et al., 2018). Researchers have found that Berberines changed gut microbiota physiology, as well as the composition and function of the bacteria. In particular, it reduced the activity of the bile salt hydrolase expressing bacteria Clostridiumspp, which altered bile acid metabolism and activated FXR signaling (Tian Y. et al., 2019). Another study found that acetylation factors and Bacteroides were increased in the intestine of mice treated with fexaramine (an FXR agonist). This process increased the secretion of taurolithocholic acid (TLCA), fibroblast growth factor 15 (FGF15) and glucagon like peptide 1 (GLP-1), which stimulated TGR5 expression in the intestine, promoted the secretion of GLP-1, improved insulin and glucose tolerance, and promoted the browning of white adipose tissue in mice (Pathak et al., 2018).

\section{Gut Microbiota Dysbiosis Can Suppress the Metabolism of Branched Chain Amino Acids}

Branched chain amino acids (BCAAs, i.e., leucine, isoleucine, and valine) are essential amino acids. They are commonly obtained from the diet, are elementary components for muscle tissue construction, and are essential amino acids for normal human activity (Wu, 2013). BCAAs are not only closely related to fatty acid synthesis, glucose transport and intestinal function regulation, but are also involved in the regulation of energy consumption and the prevention of early chronic metabolic diseases (Yoneshiro et al. 2019). Compared with the original gut microbiota composition, BCAA levels in patients with gut microbiota metabolic disorders is generally downregulated (Zeng et al., 2020). In other words, disorders of bacterial community structure inhibit the synthesis and/or release of BCAAs. Disordered gut microbiota initiated decreases in general controlled nonderepressible 2 (GCN2) kinase levels, hindered the phosphorylation of eukaryotic translation initiation factor $2 \alpha$ (eIF2 $\alpha$ ), and inhibited the biosynthesis of amino acids, thus contributing to the occurrence of endoplasmic reticulum stress. Eventually, chronic inflammation and obesity take place in the human body (Tian M. et al., 2019; Golonka et al., 2020). Additionally, other studies have shown that BACC also improved the abundance of gut microbiota. Leucine and isoleucine regulate the expression of lipid metabolism genes via SIRT1 and the lipolytic gene AMP-activated protein kinase (AMPK), increase the abundance of Lachnospiraceae, decrease the abundance of Desulfovibrionaceae and relieve lipid deposition (Ma et al., 2020). Asis reported that BCAA and gut microbiota mutually regulate obesity (Saad et al., 2016; Yue et al., 2019; Zhang L. et al., 2020): Gut microbiota affect the synthesis and metabolism of BCAA by acting on signaling pathways, but the reduction of BCAA biosynthesis will lead to chronic inflammation in the body, which can aggregate obesity indicators and cause a serious imbalance in gut microbiota proportions.

\section{Gut Microbiota Dysbiosis Can Affect the Release of Gut Hormones}

Gut hormones are peptides that are released into circulation by endocrine cells and gastrointestinal tract neurons. They not only regulate the secretion of digestive glands and alimentary canal movements, but are also responsible for the release of other hormones (Gribble and Reimann, 2019). As mentioned earlier, the final product of indigestible dietary fiber which is fermented by colon gut microbiota is SCFAs. The release of diverse intestinal 
TABLE 1 | Ginsenosides significantly improve obesity and its complications by regulating gut microbiota.

\begin{tabular}{|c|c|c|c|c|c|c|c|}
\hline Diseases & $\begin{array}{l}\text { Compounds/ } \\
\text { extracts }\end{array}$ & Subject & Gender & Period & $\begin{array}{l}\text { Main } \\
\text { gut microbiota analysis }\end{array}$ & Mechanism & References \\
\hline Obesity & Ginsenoside Rb1 & Mice & $\begin{array}{l}\text { Both } \\
\text { Genders }\end{array}$ & 4 weeks & $\begin{array}{l}\text { The abundance of Clostridia and } \\
\text { Lactococcus lactis increased }\end{array}$ & $\begin{array}{l}\text { Enhance the production of acetate } \\
\text { and butyrate contents of all SCFA }\end{array}$ & $\begin{array}{l}\text { Hasebe et al. } \\
\text { (2016) }\end{array}$ \\
\hline Obesity & Ginsenoside Rb1 & Mice & Male & 8 weeks & $\begin{array}{l}\text { The relative abundance of Firmicutes } \\
\text { phylum increased and the relative } \\
\text { abundance of Bacteroidetes phylum } \\
\text { improved }\end{array}$ & $\begin{array}{l}\text { Reduce the overall diversity of the } \\
\text { gut microbiota in feces and } \\
\text { change the microbial composition }\end{array}$ & $\begin{array}{l}\text { Bai et al. } \\
(2021)\end{array}$ \\
\hline Obesity & $\begin{array}{l}\text { Panax notoginseng } \\
\text { saponins }\end{array}$ & Mice & Male & 7 Weeks & $\begin{array}{l}\text { The abundance of Akkermansia } \\
\text { muciniphila and Parabacteroides } \\
\text { distasonis increased }\end{array}$ & $\begin{array}{l}\text { Activate leptin AMPK/STAT3 } \\
\text { signaling pathway to promote BAT } \\
\text { thermogenesis and beige } \\
\text { adipocyte reconstruction }\end{array}$ & $\begin{array}{l}\text { Xu et al. } \\
\text { (2020b) }\end{array}$ \\
\hline $\begin{array}{l}\text { Obesity } \\
\text { induced } \\
\text { colitis }\end{array}$ & Ginsenoside Rk3 & Mice & Male & 8 weeks & $\begin{array}{l}\text { The relative abundance of } \\
\text { Actinomycetes and Clostridiathat, } \\
\text { Parabacteroides, Lactobacillus, } \\
\text { Butyricicoccus and Clostridium } \\
\text { increased, while that of Akkermansia, } \\
\text { Acetobacter, Enterobacter, and } \\
\text { Anaerotruncus decreased }\end{array}$ & $\begin{array}{l}\text { Inhibit TLR4/NF- } \mathrm{BB} \text { signaling } \\
\text { pathway, and improve the } \\
\text { metabolic imbalance of intestinal } \\
\text { flora, as well as significantly reduce } \\
\text { the ratio of Firmicum/Bacteroide } \\
\text { and relieve the inflammatory } \\
\text { cascade }\end{array}$ & $\begin{array}{l}\text { Chen et al. } \\
(2021)\end{array}$ \\
\hline Diabetes & Ginsenoside T19 & Mice & Male & 6 weeks & $\begin{array}{l}\text { The value of Firmicutes/Bacteroidetes } \\
\text { decreased and the relative abundance } \\
\text { of the Lachnospiraceae family } \\
\text { remarkably raised }\end{array}$ & $\begin{array}{l}\text { Lower the levels of blood glucose } \\
\text { and lipid, alleviate insulin } \\
\text { resistance via AMPK and PI3K } \\
\text { Pathways }\end{array}$ & $\begin{array}{l}\text { Xu et al. } \\
\text { (2020a) }\end{array}$ \\
\hline Diabetes & Ginsenoside Rg5 & Mice & Male & 4 weeks & $\begin{array}{l}\text { The abundance of Firmicutes and } \\
\text { Verrucomicrobia decreased at the } \\
\text { phylum level, and the abundance of } \\
\text { Bacteroidetes and } \\
\text { Proteobacteria increased }\end{array}$ & $\begin{array}{l}\text { Repair intestinal barrier function } \\
\text { and relieve metabolic } \\
\text { endotoxemia-related inflammatory } \\
\text { pathways }\end{array}$ & $\begin{array}{l}\text { Wei et al. } \\
(2020)\end{array}$ \\
\hline Diabetes & $\begin{array}{l}\text { Ginsenoside Rb1+ } \\
\text { Ginseng } \\
\text { polysaccharides (GP) }\end{array}$ & Rats & Male & 30 days & $\begin{array}{l}\text { The abundance of } \beta \text {-D-glucosidase } \\
\text { producing probiotics Bifidobacteria spp. } \\
\text { Bacteroides spp. and Lactobacillus spp. } \\
\text { showed no significant change }\end{array}$ & $\begin{array}{l}\text { Regulate intestinal flora, improve } \\
\text { fecal } \beta \text { - D-glucosidase activity, } \\
\text { and improve the conversion rate of } \\
\text { ginsenoside Rb1 to CK }\end{array}$ & $\begin{array}{l}\text { Li et al. } \\
\text { (2018a) }\end{array}$ \\
\hline NAFLD & $\begin{array}{l}\text { Ginsenoside Rg1 + } \\
\text { Rb1 + Rg3 }\end{array}$ & Human & $\begin{array}{l}\text { Both } \\
\text { Genders }\end{array}$ & 4 weeks & $\begin{array}{l}\text { The abundance of Lactobacillus } \\
\text { significantly increased }\end{array}$ & $\begin{array}{l}\text { Improve liver enzymes (alanine } \\
\text { aminotransferase) and fatigue } \\
\text { score by modulating gut } \\
\text { microbiota }\end{array}$ & $\begin{array}{l}\text { Hong et al. } \\
(2020)\end{array}$ \\
\hline NAFLD & Ginsenoside extract & Mice & Male & 12 weeks & $\begin{array}{l}\text { The abundance of Bacteroidetes } \\
\text { significantly increased and the ratio of } \\
\text { Firmicutes to Bacteroidetes down- } \\
\text { regulated }\end{array}$ & $\begin{array}{l}\text { Enhance the gut barrier function, } \\
\text { restore the energy balance, and } \\
\text { alleviate metabolic inflammation by } \\
\text { gut microbiota regulation }\end{array}$ & $\begin{array}{l}\text { Liang et al. } \\
(2021)\end{array}$ \\
\hline
\end{tabular}

Note: Human studies in the table were randomized controlled clinical trials.

hormones is closely related to SCFA metabolism. For example, acetate enters the brain through the blood-brain barrier and blood circulation, activates the parasympathetic nervous system, promotes islet B cells to secrete large amounts of insulin, and induced glucose conversion into ATP. In addition, it promoted the release of ghrelin from the stomach, which triggered eating in order to alleviate hunger sensations. Over time, individuals with imbalanced gut microbiota continued to increase their food intake, thus increasing the risk of both insulin resistance and obesity. Peptide YY (PYY) is a kind of intestinal hormone expressed in digestive tract L cells which are released into blood circulation following eating. PYY significantly reduced food intake, which also relieved adipose deposition and improved obesity symptoms (Lafferty et al.,
2018). SCFAs increases caused by gut microbiota imbalances have been shown to combine with the actions of the GPR41 receptor to promote the secretion of PYY by terminal ileum $\mathrm{L}$ cells (Duca et al., 2013). PYY slowed down intestinal peristalsis, prolonged the time of food passing through the intestine, promoted the absorption of nutrients, and increased lipid accumulation. Therefore-as the gut microbiota affected the synthesis and release of SCFAs, its regulatory effects on intestinal hormones [(i.e., GLP-1 and cholecystokinin (CCK)], were paralleled by those of PYY (Diamant et al., 2011; Perry and Wang, 2012). The gut microbiota targeted these intestinal hormones, which therefore affected obesity occurrence. This is not only a novel factor influencing the link between obesity and abnormal gut 
microbiota composition, but also represents a potential new breakthrough for future obesity treatment.

\section{GINSENOSIDES ACT AS ANTI-OBESITY AGENTS BY MODULATING THE GUT MICROBIOTA AS A NOVEL TARGET}

The anti-obesity effects of ginsenosides are tightly connected with their regulatory role on gut microbiota. When ginsenosides are metabolized in the intestine, they significantly control the circulating levels of gut hormone metabolites that are related to obesity, improve the intestinal microenvironment, and enhance the barrier function of the intestinal tract. In recent years, several reports have suggested that ginsenosides family could improve outcomes related to obesity and its comorbidities by modulating flora functions and restoring the structure of gut microbiota (Table 1) (Li J. et al., 2018; Wei et al., 2020). This is undoubtedly a promising target for the treatment of metabolic disorders caused by obesity.

\section{Ginsenosides Have Intervention Effects on Obesity by Promoting Short Chain Fatty Acids Metabolism}

SCFAs, known as the "secret weapon" of gut microbiota, participate in the process of material absorption and lipid metabolism (Den Besten et al., 2013, 2015; Canfora et al., 2015). The dietary fiber consumed by organism is not digested directly by intestinal tract, but by SCFAs to complete digestion engineering; Meanwhile, the production of SCFAs may increase with this dietary fiber intake. SCFAs play a large role in maintaining the normal functions of the large intestine, as well as the morphology and function of colon epithelial cells. They also promote the synthesis and accumulation of lipids in hosts, which can lead to obesity once fatty acid metabolism is blocked (Kasubuchi et al., 2015; Figueiredo et al., 2017; McNabney and Henagan, 2017). Ginsenoside Rk3 administration has been demonstrated to significantly attenuate weight loss, increase SCFAs levels (including acetic acid, butyric acid and isovaleric acid), protect intestinal barrier function, and blockade the Nucleotide-binding oligomerization domain-like receptor 3 (NLRP3) inflammasome pathway (Tian et al., 2020). Fermented ginseng has also been shown to promote SCFA oxidation and decomposition, which significantly improves the capability of SCFAs to produce Akkermansia (which is in the Verrucomicrobia phylum) (Fan et al., 2019). Another study found that the conversion of ginsenoside $\mathrm{Rb} 1$ to ginsenoside compound $\mathrm{C}$ increased the number of bacterial genera (including Clostridia and Lactococcus lactis), and enhanced the acetate and butyrate contents of all SCFAs in the cecum and feces (Hasebe et al., 2016). It is worth noting that, although most studies focus on the short-chain fatty acids produced by the microbiota, long-chain fatty acids, often in the form of esters, are also important gut microbiota metabolites. It has been found that Enterotoxigenic Escherichia coli can block the uptake of long-chain fatty acids (LCFAs) into intestinal epithelial cells by activating the phosphorylation of peroxisome proliferator activated receptor $\gamma$ (PPAR- $\gamma$ ) (Li et al., 2019). Ginsenoside extract (GE) has also been shown to be capable of inducing Enterococcus faecalis to produce unsaturated LCFA and mystic acid. This action significantly improved obesity by activating brown adipose tissue (BAT) and promoting formation of beige fat (Quan et al., 2020).

\section{Intervention Effects of Ginsenosides on Obesity by Improving Inflammation Reactions}

Chronic inflammation caused by lipid accumulation is mainly mediated by LPS stimulating immune reactions and the release of pro-inflammatory factors. LPS, a component of Gram-negative bacteria in the gut, is constantly produced in the intestinal tract through the death of Gram-negative bacteria (Rietschel et al., 1994). LPS enters the plasma and is transported by lipopolysaccharide binding protein (Hersoug et al., 2018; Molinaro et al., 2020). The most general change related to LPS is the increased expression of inflammatory factors, such as IL-1, IL-6, TNF- $\alpha$, monocyte chemoattractant protein-1(MCP-1) (Seifert et al., 2015). These pro-inflammatory factors cause the structural destruction of gut microbiota, which can then induce systemic inflammation in obese individuals. Ginsenosides dramatically ameliorate obesity induced by high-fat diets via regulation of the homeostasis of gut microbiota. Research has suggested that water-extracted white ginseng (WEWG) is rich in diverse high-polar-to-low-polar ginsenosides, which are potentially more suitable for correcting obesity-related gut microbiota disorders. Specifically, WEWG reduced the ratio of Firmicutes to Bacteroidetes by $65 \%$, and significantly increased the abundance of Lactobacillus and Parabacteroides. This improved gut microbiota balance could better alleviate systemic inflammation and enteric metabolic disorders-and thus ultimately had anti-obesity effects (Zhou et al., 2020). The ginsenoside $\mathrm{Rb} 1$ has been shown to significantly decrease body weight, alleviate fasting blood glucose, improve blood lipid profiles, and decrease inflammation levels in patients when downward trends of gut microbiota diversity in fecal samples. After Rb1 consumption, Firmicutes phylum increased and the relative abundance of Bacteroidetes phylum improved. At the family level, Helicobacteraceae and Ruminococcaceae decreased and Rikenellaceae were enriched (Bai et al., 2021). In addition, in the model of obesity-induced colitis, ginsenoside Rk3 effectively ameliorated the metabolic dysbiosis of intestinal flora with significantly decreased Firmicute/Bacteroidete ratios and suppressed the inflammatory cascade by inhibiting the TLR4/ NF- $\kappa \mathrm{B}$ (Toll-like receptor 4/nuclear factor kappa-B) signaling pathway (Chen et al., 2021).

\section{Intervention Effects of Ginsenosides on Obesity via the Regulation of Gut Hormones}

In recent years, research on metabolic diseases, including obesity and diabetes, has focused on understanding gut hormones. Common intestinal hormones include ghrelin and gastrin, which are secreted from the stomach, insulin and glucagon, which are secreted from the pancreas, CCK, ghrelin, gastric inhibitory peptide (GIP) and motilin, which are secreted from the small intestine, as well as PYY and GLP-1, which are secreted 
from the large intestine. The secretion of these hormones is closely related to changes in the composition of gut microbiota. The ginsenoside $\mathrm{Rg} 3$ has been shown to stimulate GLP-1 secretion by activating sweet receptor signals, and the Ginsenoside $\operatorname{Rg} 1$ can significantly increase phosphorylation levels of AMPK in epididymal white adipose tissue and 3T3-L1 cells, which thereby inhibits adipogenesis and counters adipose accumulation (Kim et al., 2015; Liu et al., 2018). Panax notoginseng saponins (PNS), which includes multiple ginsenosides, has been demonstrated to reshape the murine gut microbiota by increasing the abundance of Akkermansia muciniphila and Parabacteroides distasonis. This stimulates the reconstruction of beige adipocytes by activating the leptinAMPK/STAT3 signaling pathway, which ultimately promotes energy expenditure. However, PNS-induced modulation of gut microbiota has negative effects in leptin gene-deficient mice, suggesting that PNS' obesity improving effects are inseparable from the interaction between intestinal flora and insulin (Xu Y. et al., 2020). Ginsenoside Rg3 is able to stimulate GLP-1 secretion by activating sweet receptor signals, and both the secretion of GLP-1 and the occurrence of inflammation may be related to the composition of gut microbiota. GLP-1 exerts anti-obesity effects by delaying gastric emptying, reducing food intake, stimulating insulin release, improving insulin sensitivity (Van Can et al., 2014), and sending appetite suppressor signals through the sympathetic nervous system. Rg1 has also been shown to significantly increase AMPK phosphorylation levels in epididymal white adipose tissue and 3T3-L1 cells. This inhibits adipogenesis, reduces intracellular lipid content, decreases adipocyte size, and counters adipose accumulation (Kim et al., 2015; Liu et al., 2018). However, there are relatively few reports on gut microbiota as an obesity-improving therapeutic target for ginsenosides, mainly because it difficult to detect specific changes in gut microbiota composition after administration. In addition, the results of different analysis methods adopted in the original data, such as $16 \mathrm{~S}$ rRNA sequencing and metagenomics, may be quite diverse, which obscures the stability of the results. Therefore, further exploration of these complex mechanism is needed to fully understand how ginsenosides improve obesity symptoms by restoring the dysbiosis of microorganisms.

\section{INTERVENTION EFFECTS OF GINSENOSIDES ON OBESITY-RELATED COMPLICATIONS}

There has been a troubling increase in the worldwide prevalence of diabetes as people's lifestyles (i.e., excessive energy intake and decreased exercise) have become altered (Balakumar et al., 2016). Diabetes is now one of the most common endocrine and metabolic diseases, which can be primarily attributed to evolving obesity and insulin resistance. Thus, diabetes is one of the most important complications of obesity. Insulin resistance and insulin secretion imbalance are the two key pathological characteristics of diabetic patients. Insulin resistance is induced by a long-term imbalance of glucose absorption and metabolism, and interacts with disordered gut microbiota compositions (Yang et al., 2018). Ginsenoside family compounds are a potentially effective drug for treating type 2 diabetes by improving gut microbiota. Ginsenoside T19 is able to effectively lower blood glucose and lipid levels, ameliorate insulin resistance, improve liver and pancreatic histopathology by decreasing Firmicutes/ Bacteroidetes values, and increasing the relative abundance of beneficial Lachnospiraceae bacteria regulate carbohydrate metabolism (Xu J. et al., 2020). Studies have shown that the ginsenoside $\operatorname{Rg} 5$ significantly relieved inflammation related to metabolic endotoxemia, and repaired the original function of the intestinal barrier. It also appears to significantly reverse gut microbiota malnutrition while reducing the Firmicutes/ Bacteroidetes ratio significantly. Additionally, $\operatorname{Rg} 5$ dramatically reduced the abundance of Firmicutes and Verrucomicrobia in diabetic mice at the phylum level, and increased the abundance of Bacteroidetes and Proteobacteria, suggesting that Rg5 can reverse gut microbiota disorders and metabolic disorders related to Type 2 diabetes (Wei et al., 2020). An investigation by Li found that the ginsenoside Rb1 had hypoglycemic activity in diabetic rat model. It worked by promoting $\beta$-Glucosidase activity, which is mainly produced by Bififidobacteria. Ginseng polysaccharides enhanced the hypoglycemic activity of ginsenoside Rb1 and altered its biotransformation pathway, which promoted the transformation of ginsenoside $\mathrm{Rb} 1$ to $\mathrm{CK}$. The improved Rb1 bioavailability contributed to hypoglycemic effects by modulating gut microbiota (Li J. et al., 2018). Another study showed that the main reason that $\mathrm{Rb} 1$ exposure increases in diabetic rats led to increased Rb1 absorption through the intestinal tract, and helped gut microbiota inhibit the de-glycosylation process, which positively affected the clinical treatment of diabetes (Liu et al., 2015). Generally, gut microbiota promotes the metabolism transformation of ginsenosides, improves their bioavailability, and stimulates the local production of secondary metabolites. Thus, they have a large role in lowering blood glucose and treating diabetes.

In addition to diabetes, ginsenosides have also been applied to treat NAFLD (Nonalcoholic fatty liver disease). In the west, $20-30 \%$ of the adult population have NAFLD (Loomba and Sanyal, 2013). One of the major causes of NAFLD is the obesity-induced accumulation of lipids in the liver, which can result in dyslipidemia and symptoms of insulin resistance. Several recent studies have found that the "gut-liver axis" has an influential role in ameliorating NAFLD (Porras et al., 2017; Carbajo-Pescador et al., 2019; Albillos et al., 2020). PNS appears to exhibit potent anti-fibrotic effects in NAFLD mice, and PNS intervention decelerates gut-to-liver translocation of microbiota-derived SCFAs products, which significantly improves the permeability of intestinal mucosa by modulating gut microbiota ( $\mathrm{Xu}$ et al., 2021). A clinical report revealed that, following administration of Korea Red Ginseng (2,000 mg/day, ginsenoside Rg1 + Rb1 + Rg3 $4.5 \mathrm{mg} / \mathrm{g}$ ) to $45 \mathrm{NAFLD}$ patients over four weeks (Hong et al., 2020), liver alanine aminotransferase levels were significantly improved, and there was a remarkable increase in the abundance of Lactobacillus. In addition, Akkermansia increased in the group treated with ginsenosides, while other liver markers, including AST, triglyceride, and $\gamma$-GT, showed significant decrease when compared with the placebo group. Another research report 
showed that GE improved the intestine's mucosal barrier, and exerted anti-inflammatory effects by restoring the composition of gut microbiota in a NAFLD mice model in a dose-dependent manner. Through network analysis, it was shown that GE inhibited $\mathrm{NF}-\kappa \mathrm{B} / \mathrm{I} \kappa \mathrm{B}$ signaling and suppressed the release of proinflammatory factors. In addition, GE's improvement effects on NAFLD were inseparable from the promotion of liver lipolysis gene (CPT-1 $\alpha)$ expression and amelioration of leptin resistance (Liang et al., 2021).

\section{DISCUSSION}

There are considerable reports that explore the underlying mechanisms of ginsenosides' anti-obesity effects. However, most of research are concentrated on describing inflammatory reactions or molecular signaling pathways, aiming to illustrate the receptors that ginsenosides activate and potential clinical targets. As an indispensable community of intestinal host, gut microbiota is highly involved in food absorption and metabolism. Because the mechanism of action of ginsenosides for obesity treatment is relatively complicated, we sum up two possible pathways that are mediated by gut microbiota. One possibility is that, in term of the low bioavailability of ginsenosides in the body, it might undergo multiple gut microbiota-mediated deglycosylation reactions in order to produce finalized ginsenosides compounds. These metabolites have beneficial effects on the treatment of obesity and its complications. Another possibility is the gut microbiome itself is a target that cannot be underestimated in the process of improving obesity. Ginsenosides are probably able to regulate the bacteria and rectify the structure of the flora, ameliorating obesity-related complications by promoting the release of related endogenous substances and regulating glucose and lipid metabolism. In summary, the mechanism by which ginsenosides regulate microbiota and improve obesity is still fairly understudied. We are not explicit in whether the effects of ginsenosides on the structure of intestinal flora are direct or indirect, or how secondary metabolites of ginsenosides may play a role in improving obesity. In view of the insufficient research on ginsenosides to alter the abundance of specific bacteria in the process of animal experiment research is not comphesive enough, and few reports on the regulation of gut microbiota by ginsenosides in clinical cases, which restricts clinical application of ginsenosides in obesity, more research should be carried out on these aspects, so as to provide new ideas for the research on the mechanism of ginsenosides improving obesity and its complications.

\section{REFERENCES}

Akao, T., Kida, H., Kanaoka, M., Hattori, M., and Kobashi, K. (1998). Intestinal Bacterial Hydrolysis Is Required for the Appearance of Compound $\mathrm{K}$ in Rat Plasma after Oral Administration of Ginsenoside Rb1 from Panax Ginseng. J. Pharm. Pharmacol. 50 (10), 1155-1160. doi:10.1111/j.20427158.1998.tb03327.x

Albillos, A., de Gottardi, A., and Rescigno, M. (2020). The Gut-Liver axis in Liver Disease: Pathophysiological Basis for Therapy. J. Hepatol. 72 (3), 558-577. doi:10.1016/j.jhep.2019.10.003

\section{CONCLUDING REMARKS}

Obesity, a chronic metabolic epidemic, has gradually become an issue throughout the world. Accumulating evidence has shown that ginsenosides have anti-obesity effects. Ginsenosides not only improve glucose metabolism, increase insulin sensitivity, and promote lipid transport, but also alleviate symptoms of obesity-related complications like diabetes and NAFLD. Gut microbiota is involved in the pathogenesis of obesity in the fountainhead step, which is responsible for the regulation of fatty acid oxidation, fatty acid synthesis, bile acid release, metabolism of branched chain amino acids, transformation of intestinal permeability, and mediation of a variety of inflammatory reactions. The interaction between ginsenosides and gut microbiota may have far-reaching impacts that improve obesity.

Although targeting microflora with ginsenosides is a technique currently in its infancy, it has the potential to become a clinical choice for the treatment of obesity and other metabolic diseases if thoroughly researched. In the present review, we described several potential pathological mechanisms that could affect gut microbiota and lead to obesity. Next, wanting to further verify that ginsenosides had competent anti-obesity effects, we explored the rationality and necessity of gut microbiota as a novel treatment target. To this end, further research evaluating the feasibility of gut microbiota as a novel target of ginsenosides that has implications for curing obesity and related complications is needed.

\section{AUTHOR CONTRIBUTIONS}

TZ: Writing-Original Draft and Visualization; WL: Writing-Review and Editing; Z-tW, LY and MZ: Conceptualization and Supervision; LDing: Conceptualization, Writing-Review and Editing and Supervision.

\section{FUNDING}

This work is financially sponsored by the Natural Science Foundations of China to LD (81773961) and ZW (81920108033), China Postdoctoral Science Foundation (2020M681368) to WL as well as Innovation Project for Postgraduates of Shanghai University of Traditional Chinese Medicine (Grant Number: Y2021032) to TZ.

Alex, S., Lange, K., Amolo, T., Grinstead, J. S., Haakonsson, A. K., Szalowska, E., et al. (2013). Short-chain Fatty Acids Stimulate Angiopoietin-like 4 Synthesis in Human colon Adenocarcinoma Cells by Activating Peroxisome Proliferator-Activated Receptor $\gamma$. Mol. Cel. Biol. 33 (7), 1303-1316. doi:10.1128/mcb.00858-12

An, R., Shen, J., Bullard, T., Han, Y., Qiu, D., and Wang, S. (2020). A Scoping Review on Economic Globalization in Relation to the Obesity Epidemic. Obes. Rev. 21 (3), e12969. doi:10.1111/obr.12969

Ang, Z., and Ding, J. L. (2016). GPR41 and GPR43 in Obesity and Inflammation Protective or Causative? Front. Immunol. 7, 28. doi:10.3389/fimmu.2016.00028 Argentato, P. P., de Cássia César, H., Estadella, D., and Pisani, L. P. (2018). Programming Mediated by Fatty Acids Affects Uncoupling Protein 1 (UCP-1) 
in Brown Adipose Tissue. Br. J. Nutr. 120 (6), 619-627. doi:10.1017/ S0007114518001629

Aronsson, L., Huang, Y., Parini, P., Korach-André, M., Håkansson, J., Gustafsson, J. A., et al. (2010). Decreased Fat Storage by Lactobacillus Paracasei Is Associated with Increased Levels of Angiopoietin-like 4 Protein (ANGPTL4). PLoS One 5 (9), e13087. doi:10.1371/journal.pone.0013087

Bäckhed, F., Ding, H., Wang, T., Hooper, L. V., Koh, G. Y., Nagy, A., et al. (2004). The Gut Microbiota as an Environmental Factor that Regulates Fat Storage. Proc. Natl. Acad. Sci. U. S. A. 101 (44), 15718-15723. doi:10.1073/ pnas.0407076101

Bai, Y., Bao, X., Mu, Q., Fang, X., Zhu, R., Liu, C., et al. (2021). Ginsenoside Rb1, Salvianolic Acid B and Their Combination Modulate Gut Microbiota and Improve Glucolipid Metabolism in High-Fat Diet Induced Obese Mice. PeerJ 9, e10598. doi:10.7717/peerj.10598

Balakumar, P., Maung-U, K., and Jagadeesh, G. (2016). Prevalence and Prevention of Cardiovascular Disease and Diabetes Mellitus. Pharmacol. Res. 113 (Pt A), 600-609. doi:10.1016/j.phrs.2016.09.040

Behr, C., Slopianka, M., Haake, V., Strauss, V., Sperber, S., Kamp, H., et al. (2019). Analysis of Metabolome Changes in the Bile Acid Pool in Feces and Plasma of Antibiotic-Treated Rats. Toxicol. Appl. Pharmacol. 363, 79-87. doi:10.1016/ j.taap.2018.11.012

Bernard, A., Ancel, D., Passilly-Degrace, P., Landrier, J. F., Lagrost, L., and Besnard, P. (2019). A Chronic LPS-Induced Low-Grade Inflammation Fails to Reproduce in Lean Mice the Impairment of Preference for Oily Solution Found in Diet-Induced Obese Mice. Biochimie 159, 112-121. doi:10.1016/j.biochi.2018.08.004

Boutens, L., Hooiveld, G. J., Dhingra, S., Cramer, R. A., Netea, M. G., and Stienstra, R. (2018). Unique Metabolic Activation of Adipose Tissue Macrophages in Obesity Promotes Inflammatory Responses. Diabetologia 61 (4), 942-953. doi:10.1007/s00125-017-4526-6

Brooks-Worrell, B. M., and Palmer, J. P. (2019). Setting the Stage for Islet Autoimmunity in Type 2 Diabetes: Obesity-Associated Chronic Systemic Inflammation and Endoplasmic Reticulum (ER) Stress. Diabetes Care 42 (12), 2338-2346. doi:10.2337/dc19-0475

Canfora, E. E., Jocken, J. W., and Blaak, E. E. (2015). Short-chain Fatty Acids in Control of Body Weight and Insulin Sensitivity. Nat. Rev. Endocrinol. 11 (10), 577-591. doi:10.1038/nrendo.2015.128

Cani, P. D., Amar, J., Iglesias, M. A., Poggi, M., Knauf, C., Bastelica, D., et al. (2007). Metabolic Endotoxemia Initiates Obesity and Insulin Resistance. Diabetes 56 (7), 1761-1772. doi:10.2337/db06-1491

Cani, P. D., Possemiers, S., Van De Wiele, T., Guiot, Y., Everard, A., Rottier, O., et al. (2009). Changes in Gut Microbiota Control Inflammation in Obese Mice through a Mechanism Involving GLP-2-Driven Improvement of Gut Permeability. Gut 58 (8), 1091-1103. doi:10.1136/gut.2008.165886

Cani, P. D., Bibiloni, R., Knauf, C., Waget, A., Neyrinck, A. M., Delzenne, N. M., et al. (2008). Changes in Gut Microbiota Control Metabolic EndotoxemiaInduced Inflammation in High-Fat Diet-Induced Obesity and Diabetes in Mice. Diabetes 57 (6), 1470-1481. doi:10.2337/db07-1403.Additional

Carbajo-Pescador, S., Porras, D., García-Mediavilla, M. V., Martínez-Flórez, S., Juarez-Fernández, M., Cuevas, M. J., et al. (2019). Beneficial Effects of Exercise on Gut Microbiota Functionality and Barrier Integrity, and Gut-Liver Crosstalk in an In Vivo Model of Early Obesity and Non-alcoholic Fatty Liver Disease. Dis. Model. Mech. 12 (5), dmm039206. doi:10.1242/dmm.039206

Carvalho, B. M., Guadagnini, D., Tsukumo, D. M. L., Schenka, A. A., Latuf-Filho, P., Vassallo, J., et al. (2012). Modulation of Gut Microbiota by Antibiotics Improves Insulin Signalling in High-Fat Fed Mice. Diabetologia 55 (10), 2823-2834. doi:10.1007/s00125-012-2648-4

Chávez-Talavera, O., Tailleux, A., Lefebvre, P., and Staels, B. (2017). Bile Acid Control of Metabolism and Inflammation in Obesity, Type 2 Diabetes, Dyslipidemia, and Nonalcoholic Fatty Liver Disease. Gastroenterology 152 (7), 1679-e3. doi:10.1053/j.gastro.2017.01.055

Chen, H., Yang, H., Deng, J., and Fan, D. (2021). Ginsenoside Rk3 Ameliorates Obesity-Induced Colitis by Regulating of Intestinal Flora and the TLR4/NF-Kb Signaling Pathway in C57BL/6 Mice. J. Agric. Food Chem. 69 (10), 3082-3093. doi:10.1021/acs.jafc.0c07805

Chiang, J. Y. L. (2017). Bile Acid Metabolism and Signaling in Liver Disease and Therapy. Liver Res. 1 (1), 3-9. doi:10.1016/j.livres.2017.05.001

Cho, Y. H., Ahn, S. C., Lee, S. Y., Jeong, D. W., Choi, E. J., Kim, Y. J., et al. (2013). Effect of Korean Red Ginseng on Insulin Sensitivity in Non-diabetic Healthy
Overweight and Obese Adults. Asia Pac. J. Clin. Nutr. 22 (3), 365-371. doi:10.6133/apjen.2013.22.3.04

Choi, K. T. (2008). Botanical Characteristics, Pharmacological Effects and Medicinal Components of Korean Panax Ginseng C A Meyer. Acta Pharmacol. Sin. 29 (9), 1109-1118. doi:10.1111/j.1745-7254.2008.00869.x

David, L. A., Maurice, C. F., Carmody, R. N., Gootenberg, D. B., Button, J. E., Wolfe, B. E., et al. (2014). Diet Rapidly and Reproducibly Alters the Human Gut Microbiome. Nature 505 (7484), 559-563. doi:10.1038/nature12820

De la Cuesta-Zuluaga, J., Mueller, N., Álvarez-Quintero, R., Velásquez-Mejía, E., Sierra, J., Corrales-Agudelo, V., et al. (2019). Higher Fecal Short-Chain Fatty Acid Levels Are Associated with Gut Microbiome Dysbiosis, Obesity, Hypertension and Cardiometabolic Disease Risk Factors. Nutrients 11 (1), 51. doi:10.3390/nu11010051

De Lartigue, G., Barbier de la Serre, C., Espero, E., Lee, J., and Raybould, H. E. (2011). Diet-induced Obesity Leads to the Development of Leptin Resistance in Vagal Afferent Neurons. Am. J. Physiol. Endocrinol. Metab. 301 (1), E187-E195. doi:10.1152/ajpendo.00056.2011

Den Besten, G., Bleeker, A., Gerding, A., Van Eunen, K., Havinga, R., Van Dijk, T. H., et al. (2015). Short-Chain Fatty Acids Protect against High-Fat DietInduced Obesity via a PPAR $\gamma$-dependent Switch from Lipogenesis to Fat Oxidation. Diabetes 64 (7), 2398-2408. doi:10.2337/db14-1213

Den Besten, G., Van Eunen, K., Groen, A. K., Venema, K., Reijngoud, D. J., and Bakker, B. M. (2013). The Role of Short-Chain Fatty Acids in the Interplay between Diet, Gut Microbiota, and Host Energy Metabolism. J. Lipid Res. 54 (9), 2325-2340. doi:10.1194/jlr.R036012

Devaraj, S., Hemarajata, P., and Versalovic, J. (2013). The Human Gut Microbiome and Body Metabolism: Implications for Obesity and Diabetes. Clin. Chem. 59 (4), 617-628. doi:10.1373/clinchem.2012.187617

Diamant, M., Blaak, E. E., and de Vos, W. M. (2011). Do nutrient-gutmicrobiota Interactions Play a Role in Human Obesity, Insulin Resistance and Type 2 Diabetes? Obes. Rev. 12 (4), 272-281. doi:10.1111/ j.1467-789X.2010.00797.x

Duca, F. A., Swartz, T. D., Sakar, Y., and Covasa, M. (2013). Decreased Intestinal Nutrient Response in Diet-Induced Obese Rats: Role of Gut Peptides and Nutrient Receptors. Int. J. Obes. (Lond) 37 (3), 375-381. doi:10.1038/ijo.2012.45

Everard, A., Belzer, C., Geurts, L., Ouwerkerk, J. P., Druart, C., Bindels, L. B., et al. (2013). Cross-talk between Akkermansia Muciniphila and Intestinal Epithelium Controls Diet-Induced Obesity. Proc. Natl. Acad. Sci. U. S. A. 110 (22), 9066-9071. doi:10.1073/pnas.1219451110

Fan, J., Wang, Y., You, Y., Ai, Z., Dai, W., Piao, C., et al. (2019). Fermented Ginseng Improved Alcohol Liver Injury in Association with Changes in the Gut Microbiota of Mice. Food Funct. 10 (9), 5566-5573. doi:10.1039/c9fo01415b

Gentile, C. L., and Weir, T. L. (2018). The Gut Microbiota at the Intersection of Diet and Human Health. Science 362 (6416), 776-780. doi:10.1126/ science.aau5812

Golonka, R. M., Xiao, X., Abokor, A. A., Joe, B., and Vijay-Kumar, M. (2020). Altered Nutrient Status Reprograms Host Inflammation and Metabolic Health via Gut Microbiota. J. Nutr. Biochem. 80, 108360. doi:10.1016/ j.jnutbio.2020.108360

Gribble, F. M., and Reimann, F. (2019). Function and Mechanisms of Enteroendocrine Cells and Gut Hormones in Metabolism. Nat. Rev. Endocrinol. 15 (4), 226-237. doi:10.1038/s41574-019-0168-8

Guo, S., Al-Sadi, R., Said, H. M., and Ma, T. Y. (2013). Lipopolysaccharide Causes an Increase in Intestinal Tight junction Permeability In Vitro and In Vivo by Inducing Enterocyte Membrane Expression and Localization of TLR-4 and CD14. Am. J. Pathol. 182 (2), 375-387. doi:10.1016/j.ajpath.2012.10.014

Guo, X., Ran, C., Zhang, Z., He, S., Jin, M., and Zhou, Z. (2017). The GrowthPromoting Effect of Dietary Nucleotides in Fish Is Associated with an Intestinal Microbiota-Mediated Reduction in Energy Expenditure. J. Nutr. 147 (5), 781-788. doi:10.3945/jn.116.245506

Hammarstedt, A., Gogg, S., Hedjazifar, S., Nerstedt, A., and Smith, U. (2018). Impaired Adipogenesis and Dysfunctional Adipose Tissue in Human Hypertrophic Obesity. Physiol. Rev. 98 (4), 1911-1941. doi:10.1152/ physrev.00034.2017

Hasebe, T., Ueno, N., Musch, M. W., Nadimpalli, A., Kaneko, A., Kaifuchi, N., et al. (2016). Daikenchuto (TU-100) Shapes Gut Microbiota Architecture and Increases the Production of Ginsenoside Metabolite Compound $\mathrm{K}$. Pharmacol. Res. Perspect. 4 (1), e00215. doi:10.1002/prp2.215 
Hersoug, L. G., Møller, P., and Loft, S. (2016). Gut Microbiota-Derived Lipopolysaccharide Uptake and Trafficking to Adipose Tissue: Implications for Inflammation and Obesity. Obes. Rev. 17 (4), 297-312. doi:10.1111/ obr. 12370

Hersoug, L. G., Møller, P., and Loft, S. (2018). Role of Microbiota-Derived Lipopolysaccharide in Adipose Tissue Inflammation, Adipocyte Size and Pyroptosis during Obesity. Nutr. Res. Rev. 31 (2), 153-163. doi:10.1017/ S0954422417000269

Hong, J. T., Lee, M. J., Yoon, S. J., Shin, S. P., Bang, C. S., Baik, G. H., et al. (2021). Effect of Korea Red Ginseng on Nonalcoholic Fatty Liver Disease: an Association of Gut Microbiota with Liver Function. J. Ginseng Res. 45 (2), 316-324. doi:10.1016/j.jgr.2020.07.004

Huang, B. Z., Tsilidis, K. K., Smith, M. W., Hoffman-Bolton, J., Visvanathan, K., Platz, E. A., et al. (2018). Polymorphisms in Genes Related to Inflammation and Obesity and Colorectal Adenoma Risk. Mol. Carcinog. 57 (10), 1278-1288. doi:10.1002/mc.22842

Jadhav, K., Xu, Y., Xu, Y., Li, Y., Xu, J., Zhu, Y., et al. (2018). Reversal of Metabolic Disorders by Pharmacological Activation of Bile Acid Receptors TGR5 and FXR. Mol. Metab. 9, 131-140. doi:10.1016/ j.molmet.2018.01.005

Jiao, A., Yu, B., He, J., Yu, J., Zheng, P., Luo, Y., et al. (2020). Short Chain Fatty Acids Could Prevent Fat Deposition in Pigs via Regulating Related Hormones and Genes. Food Funct. 11 (2), 1845-1855. doi:10.1039/ c9fo02585e

Jung, U. J., and Choi, M. S. (2014). Obesity and its Metabolic Complications: The Role of Adipokines and the Relationship between Obesity, Inflammation, Insulin Resistance, Dyslipidemia and Nonalcoholic Fatty Liver Disease. Int. J. Mol. Sci. 15 (4), 6184-6223. doi:10.3390/ijms15046184

Karam, B. S., Chavez-Moreno, A., Koh, W., Akar, J. G., and Akar, F. G. (2017). Oxidative Stress and Inflammation as central Mediators of Atrial Fibrillation in Obesity and Diabetes. Cardiovasc. Diabetol. 16 (1), 120. doi:10.1186/s12933017-0604-9

Kasubuchi, M., Hasegawa, S., Hiramatsu, T., Ichimura, A., and Kimura, I. (2015). Dietary Gut Microbial Metabolites, Short-Chain Fatty Acids, and Host Metabolic Regulation. Nutrients 7 (4), 2839-2849. doi:10.3390/ nu7042839

Keskitalo, A., Munukka, E., Toivonen, R., Hollmén, M., Kainulainen, H., Huovinen, P., et al. (2018). Enterobacter cloacae Administration Induces Hepatic Damage and Subcutaneous Fat Accumulation in High-Fat Diet Fed Mice. PLoS One 13 (5), e0198262. doi:10.1371/journal.pone.0198262

Kim, D., Kim, M., Raña, G., and Han, J. (2018a). Seasonal Variation and Possible Biosynthetic Pathway of Ginsenosides in Korean Ginseng Panax Ginseng Meyer. Molecules 23 (7), 1824. doi:10.3390/molecules23071824

Kim, H. J., Lee, S. Y., and Kim, C. M. (2018b). Association between Gene Polymorphisms and Obesity and Physical Fitness in Korean Children. Biol. Sport 35 (1), 21-27. doi:10.5114/biolsport.2018.70748

Kim, J. H., Yi, Y. S., Kim, M. Y., and Cho, J. Y. (2017). Role of Ginsenosides, the Main Active Components of Panax Ginseng, in Inflammatory Responses and Diseases. J. Ginseng Res. 41 (4), 435-443. doi:10.1016/j.jgr.2016.08.004

Kim, Y. J., Zhang, D., and Yang, D. C. (2015). Biosynthesis and Biotechnological Production of Ginsenosides. Biotechnol. Adv. 33 (6 Pt 1), 717-735. doi:10.1016/ j.biotechadv.2015.03.001

Kobyliak, N., Virchenko, O., and Falalyeyeva, T. (2016). Pathophysiological Role of Host Microbiota in the Development of Obesity. Nutr. J. 15, 43. doi:10.1186/ s12937-016-0166-9

Kootte, R. S., Levin, E., Salojärvi, J., Smits, L. P., Hartstra, A. V., Udayappan, S. D., et al. (2017). Improvement of Insulin Sensitivity after Lean Donor Feces in Metabolic Syndrome Is Driven by Baseline Intestinal Microbiota Composition. Cell Metab 26 (4), 611-e6. doi:10.1016/j.cmet.2017.09.008

Krumbeck, J. A., Rasmussen, H. E., Hutkins, R. W., Clarke, J., Shawron, K., Keshavarzian, A., et al. (2018). Probiotic Bifidobacterium Strains and Galactooligosaccharides Improve Intestinal Barrier Function in Obese Adults but Show No Synergism when Used Together as Synbiotics. Microbiome 6 (1), 121. doi:10.1186/s40168-018-0494-4

Kuhn, K. A., Schulz, H. M., Regner, E. H., Severs, E. L., Hendrickson, J. D., Mehta, G., et al. (2018). Bacteroidales Recruit IL-6-producing Intraepithelial Lymphocytes in the colon to Promote Barrier Integrity. Mucosal Immunol. 11 (2), 357-368. doi:10.1038/mi.2017.55
Lafferty, R. A., Flatt, P. R., and Irwin, N. (2018). Emerging Therapeutic Potential for Peptide YY for Obesity-Diabetes. Peptides 100, 269-274. doi:10.1016/ j.peptides.2017.11.005

Leoni, S., Tovoli, F., Napoli, L., Serio, I., Ferri, S., and Bolondi, L. (2018). Current Guidelines for the Management of Non-alcoholic Fatty Liver Disease: A Systematic Review with Comparative Analysis. World J. Gastroenterol. 24 (30), 3361-3373. doi:10.3748/wjg.v24.i30.3361

Ley, R. E., Bäckhed, F., Turnbaugh, P., Lozupone, C. A., Knight, R. D., and Gordon, J. I. (2005). Obesity Alters Gut Microbial Ecology. Proc. Natl. Acad. Sci. U. S. A. 102 (31), 11070-11075. doi:10.1073/pnas.0504978102

Ley, R. E., Turnbaugh, P. J., Klein, S., and Gordon, J. I. (2006). Microbial Ecology: Human Gut Microbes Associated with Obesity. Nature 444 (7122), 1022-1023. doi:10.1038/4441022a

Li, J., Li, R., Li, N., Zheng, F., Dai, Y., Ge, Y., et al. (2018a). Mechanism of Antidiabetic and Synergistic Effects of Ginseng Polysaccharide and Ginsenoside Rb1 on Diabetic Rat Model. J. Pharm. Biomed. Anal. 158, 451-460. doi:10.1016/ j.jpba.2018.06.024

Li, R., Zhu, N., Zhao, H., Wang, N., Sun, H., Yue, H., et al. (2018b). Effects of Ginseng Polysaccharides on the Metabolism of Ginsenoside Re In Vivo and Transformation of Ginsenoside Re In Vitro. Gaodeng Xuexiao Huaxue Xuebao/ chemical J. Chin. Univ. 39 (10), 2192-2197. doi:10.7503/cjcu20180080

Li, Z., Liu, H., Xu, B., and Wang, Y. (2019). Enterotoxigenic Escherichia coli Interferes FATP4-dependent Long-Chain Fatty Acid Uptake of Intestinal Epithelial Enterocytes via Phosphorylation of ERK1/2-Ppar $\gamma$ Pathway. Front. Physiol. 10, 798. doi:10.3389/fphys.2019.00798

Liang, W., Zhou, K., Jian, P., Chang, Z., Zhang, Q., Liu, Y., et al. (2021). Ginsenosides Improve Nonalcoholic Fatty Liver Disease via Integrated Regulation of Gut Microbiota, Inflammation and Energy Homeostasis. Front. Pharmacol. 12, 622841. doi:10.3389/fphar.2021.622841

Liu, C., Hu, M., Guo, H., Zhang, M., Zhang, J., Li, F., et al. (2015). Combined Contribution of Increased Intestinal Permeability and Inhibited Deglycosylation of Ginsenoside $\mathrm{Rb} 1$ in the Intestinal Tract to the Enhancement of Ginsenoside Rb1 Exposure in Diabetic Rats after Oral Administration. Drug Metab. Dispos. 43 (11), 1702-1710. doi:10.1124/ dmd.115.064881

Liu, H., Wang, J., Liu, M., Zhao, H., Yaqoob, S., Zheng, M., et al. (2018). Antiobesity Effects of Ginsenoside Rg1 on 3T3-L1 Preadipocytes and High Fat DietInduced Obese Mice Mediated by AMPK. Nutrients 10 (7), 830. doi:10.3390/nu10070830

Liu, Y., Deng, J., and Fan, D. (2019). Ginsenoside Rk3 Ameliorates High-Fat-Diet/ streptozocin Induced Type 2 Diabetes Mellitus in Mice via the AMPK/Akt Signaling Pathway. Food Funct. 10 (5), 2538-2551. doi:10.1039/c9fo00095j

Loomba, R., and Sanyal, A. J. (2013). The Global NAFLD Epidemic. Nat. Rev. Gastroenterol. Hepatol. 10 (11), 686-690. doi:10.1038/nrgastro.2013.171

Lu, Y., Fan, C., Li, P., Lu, Y., Chang, X., and Qi, K. (2016). Short Chain Fatty Acids Prevent High-Fat-Diet-Induced Obesity in Mice by Regulating G ProteinCoupled Receptors and Gut Microbiota. Sci. Rep. 6, 37589. doi:10.1038/ srep37589

Ma, Q. Q., Wang, G. H., Zhou, X. B., Zhu, J. L., Yue, Z. Y., and Shan, A. S. (2020). Effect and Mechanism of Leucine and Isoleucine on Fat Deposition. J. Northeast. Agric. Univ. 51 (1), 50-56. doi:10.19720/j.cnki.issn.10059369.2020.01.00

Martens, E. C., Neumann, M., and Desai, M. S. (2018). Interactions of Commensal and Pathogenic Microorganisms with the Intestinal Mucosal Barrier. Nat. Rev. Microbiol. 16 (8), 457-470. doi:10.1038/s41579-018-0036-x

McNabney, S. M., and Henagan, T. M. (2017). Short Chain Fatty Acids in the colon and Peripheral Tissues: A Focus on Butyrate, colon Cancer, Obesity and Insulin Resistance. Nutrients 9 (12), 1348. doi:10.3390/nu9121348

Mei, X., Zhang, X., Wang, Z., Gao, Z., Liu, G., Hu, H., et al. (2016). Insulin Sensitivity-Enhancing Activity of Phlorizin Is Associated with Lipopolysaccharide Decrease and Gut Microbiota Changes in Obese and Type 2 Diabetes (Db/db) Mice. J. Agric. Food Chem. 64 (40), 7502-7511. doi:10.1021/acs.jafc.6b03474

Molinaro, A., Koh, A., Wu, H., Schoeler, M., Faggi, M. I., Carreras, A., et al. (2020). Hepatic Expression of Lipopolysaccharide-Binding Protein (Lbp) Is Induced by the Gut Microbiota through Myd88 and Impairs Glucose Tolerance in Mice Independent of Obesity. Mol. Metab. 37, 100997. doi:10.1016/ j.molmet.2020.100997 
Mu, Q., Zuo, J., Zhao, D., Zhou, X., Hua, J., Bai, Y., et al. (2021). Ginsenoside Rg3 Reduces Body Weight by Regulating Fat Content and browning in Obese Mice. J. Traditional Chin. Med. Sci. 8 (1), 65-71. doi:10.1016/j.jtcms.2021.01.009

O’Reilly, M. E., Kajani, S., Ralston, J. C., Lenighan, Y. M., Roche, H. M., and McGillicuddy, F. C. (2019). Nutritionally Derived Metabolic Cues Typical of the Obese Microenvironment Increase Cholesterol Efflux Capacity of Adipose Tissue Macrophages. Mol. Nutr. Food Res. 63 (2), e1800713. doi:10.1002/ mnfr.201800713

Oliveira, L. P., Guimarães, V. H. D., Oliveira, J. R., Guimarães, A. L. S., de Paula, A. M. B., Bader, M., et al. (2020). Genetic Deletion of the Angiotensin-(1-7) Receptor Mas Leads to Alterations in Gut Villi Length Modulating TLR4/PI3K/ AKT and Produces Microbiome Dysbiosis. Neuropeptides 82, 102056. doi:10.1016/j.npep.2020.102056

Pan, W., Xue, B., Yang, C., Miao, L., Zhou, L., Chen, Q., et al. (2018). Biopharmaceutical Characters and Bioavailability Improving Strategies of Ginsenosides. Fitoterapia 129, 272-282. doi:10.1016/j.fitote.2018.06.001

Park, H. J., Kim, J. H., and Shim, I. (2019). Anti-obesity Effects of Ginsenosides in High-Fat Diet-Fed Rats. Chin. J. Integr. Med. 25 (12), 895-901. doi:10.1007/ s11655-019-3200-x

Pathak, P., Xie, C., Nichols, R. G., Ferrell, J. M., Boehme, S., Krausz, K. W., et al. (2018). Intestine Farnesoid X Receptor Agonist and the Gut Microbiota Activate G-Protein Bile Acid Receptor-1 Signaling to Improve Metabolism. Hepatology 68 (4), 1574-1588. doi:10.1002/hep.29857

Perry, B., and Wang, Y. (2012). Appetite Regulation and Weight Control: The Role of Gut Hormones. Nutr. Diabetes 2 (1), e26. doi:10.1038/nutd.2011.21

Pickard, J. M., Zeng, M. Y., Caruso, R., and Núñez, G. (2017). Gut Microbiota: Role in Pathogen Colonization, Immune Responses, and Inflammatory Disease. Immunol. Rev. 279 (1), 70-89. doi:10.1111/imr.12567

Pontarollo, G., Mann, A., Brandão, I., Malinarich, F., Schöpf, M., and Reinhardt, C. (2020). Protease-activated Receptor Signaling in Intestinal Permeability Regulation. FEBS J. 287 (4), 645-658. doi:10.1111/febs.15055

Porras, D., Nistal, E., Martínez-Flórez, S., Pisonero-Vaquero, S., Olcoz, J. L., Jover, R., et al. (2017). Protective Effect of Quercetin on High-Fat Diet-Induced Nonalcoholic Fatty Liver Disease in Mice Is Mediated by Modulating Intestinal Microbiota Imbalance and Related Gut-Liver axis Activation. Free Radic. Biol. Med. 102, 188-202. doi:10.1016/j.freeradbiomed.2016.11.037

Quan, L. H., Zhang, C., Dong, M., Jiang, J., Xu, H., Yan, C., et al. (2020). Myristoleic Acid Produced by Enterococci Reduces Obesity through Brown Adipose Tissue Activation. Gut 69 (7), 1239-1247. doi:10.1136/gutjnl-2019-319114

Reeds, D. N., Patterson, B. W., Okunade, A., Holloszy, J. O., Polonsky, K. S., and Klein, S. (2011). Ginseng and Ginsenoside Re Do Not Improve $\beta$-cell Function or Insulin Sensitivity in Overweight and Obese Subjects with Impaired Glucose Tolerance or Diabetes. Diabetes Care 34 (5), 1071-1076. doi:10.2337/dc10-2299

Regner, E. H., Ohri, N., Stahly, A., Gerich, M. E., Fennimore, B. P., Ir, D., et al. (2018). Functional Intraepithelial Lymphocyte Changes in Inflammatory Bowel Disease and Spondyloarthritis Have Disease Specific Correlations with Intestinal Microbiota. Arthritis Res. Ther. 20 (1), 149. doi:10.1186/s13075018-1639-3

Rietschel, E. T., Kirikae, T., Schade, F. U., Mamat, U., Schmidt, G., Loppnow, H., et al. (1994). Bacterial Endotoxin: Molecular Relationships of Structure to Activity and Function. FASEB J. 8 (2), 217-225. doi:10.1096/fasebj.8.2.8119492

Roh, E., Hwang, H. J., Kim, J. W., Hong, S. H., Kim, J. A., Lee, Y. B., et al. (2020). Ginsenoside Mcl Improves Liver Steatosis and Insulin Resistance by Attenuating ER Stress. J. Ethnopharmacol. 259, 112927. doi:10.1016/ j.jep.2020.112927

Ruiz-Ojeda, F. J., Méndez-Gutiérrez, A., Aguilera, C. M., and Plaza-Díaz, J. (2019). Extracellular Matrix Remodeling of Adipose Tissue in Obesity and Metabolic Diseases. Int. J. Mol. Sci. 20 (19), 4888. doi:10.3390/ijms20194888

Saad, M. J., Santos, A., and Prada, P. O. (2016). Linking Gut Microbiota and Inflammation to Obesity and Insulin Resistance. Physiology (Bethesda) 31 (4), 283-293. doi:10.1152/physiol.00041.2015

Scott, K. P., Gratz, S. W., Sheridan, P. O., Flint, H. J., and Duncan, S. H. (2013). The Influence of Diet on the Gut Microbiota. Pharmacol. Res. 69 (1), 52-60. doi:10.1016/j.phrs.2012.10.020

Seifert, L., Deutsch, M., Alothman, S., Alqunaibit, D., Werba, G., Pansari, M., et al. (2015). Dectin-1 Regulates Hepatic Fibrosis and Hepatocarcinogenesis by Suppressing TLR4 Signaling Pathways. Cell Rep 13 (9), 1909-1921. doi:10.1016/j.celrep.2015.10.058
Shibata, S. (2001). Chemistry and Cancer Preventing Activities of Ginseng Saponins and Some Related Triterpenoid Compounds. J. Korean Med. Sci. 16 Suppl (Suppl. l), S28-S37. doi:10.3346/jkms.2001.16.S.S28

Silva Figueiredo, P., Carla Inada, A., Marcelino, G., Maiara Lopes Cardozo, C., de Cássia Freitas, K., de Cássia Avellaneda Guimarães, R., et al. (2017). Fatty Acids Consumption: The Role Metabolic Aspects Involved in Obesity and its Associated Disorders. Nutrients 9 (10), 1158. doi:10.3390/nu9101158

Sonnenburg, J. L., and Bäckhed, F. (2016). Diet-microbiota Interactions as Moderators of Human Metabolism. Nature 535 (7610), 56-64. doi:10.1038/ nature 18846

Sun, H., Yang, Z., and Ye, Y. (2006). Structure and Biological Activity of Protopanaxatriol-type Saponins from the Roots of Panax Notoginseng. Int. Immunopharmacol. 6 (1), 14-25. doi:10.1016/j.intimp.2005.07.003

Tan, J., McKenzie, C., Potamitis, M., Thorburn, A. N., Mackay, C. R., and Macia, L. (2014). The Role of Short-Chain Fatty Acids in Health and Disease. Adv. Immunol. 121, 91-119. doi:10.1016/B978-0-12-800100-4.00003-9

Tate, J., Knuiman, M., Davis, W. A., Davis, T. M. E., and Bruce, D. G. (2020). A Comparison of Obesity Indices in Relation to Mortality in Type 2 Diabetes: the Fremantle Diabetes Study. Diabetologia 63 (3), 528-536. doi:10.1007/s00125019-05057-8

Tian, M., Heng, J., Song, H., Shi, K., Lin, X., Chen, F., et al. (2019a). Dietary Branched-Chain Amino Acids Regulate Food Intake Partly through Intestinal and Hypothalamic Amino Acid Receptors in Piglets. J. Agric. Food Chem. 67 (24), 6809-6818. doi:10.1021/acs.jafc.9b02381

Tian, M., Ma, P., Zhang, Y., Mi, Y., and Fan, D. (2020). Ginsenoside Rk3 Alleviated DSS-Induced Ulcerative Colitis by Protecting colon Barrier and Inhibiting NLRP3 Inflammasome Pathway. Int. Immunopharmacol. 85, 106645. doi:10.1016/j.intimp.2020.106645

Tian, Y., Cai, J., Gui, W., Nichols, R. G., Koo, I., Zhang, J., et al. (2019b). Berberine Directly Affects the Gut Microbiota to Promote Intestinal Farnesoid X Receptor Activation. Drug Metab. Dispos. 47 (2), 86-93. doi:10.1124/dmd.118.083691

Torres, S., Fabersani, E., Marquez, A., and Gauffin-Cano, P. (2019). Adipose Tissue Inflammation and Metabolic Syndrome. The Proactive Role of Probiotics. Eur. J. Nutr. 58 (1), 27-43. doi:10.1007/s00394-018-1790-2

Torres-Fuentes, C., Schellekens, H., Dinan, T. G., and Cryan, J. F. (2017). The Microbiota-Gut-Brain axis in Obesity. Lancet Gastroenterol. Hepatol. 2 (10), 747-756. doi:10.1016/S2468-1253(17)30147-4

Tsai, F., and Coyle, W. J. (2009). The Microbiome and Obesity: Is Obesity Linked to Our Gut flora? Curr. Gastroenterol. Rep. 11 (4), 307-313. doi:10.1007/s11894009-0045-z

Van Can, J., Sloth, B., Jensen, C. B., Flint, A., Blaak, E. E., and Saris, W. H. (2014). Effects of the Once-Daily GLP-1 Analog Liraglutide on Gastric Emptying, Glycemic Parameters, Appetite and Energy Metabolism in Obese, Non-diabetic Adults. Int. J. Obes. (Lond) 38 (6), 784-793. doi:10.1038/ijo.2013.162

Wang, B., Kong, Q., Li, X., Zhao, J., Zhang, H., Chen, W., et al. (2020). A High-Fat Diet Increases Gut Microbiota Biodiversity and Energy Expenditure Due to Nutrient Difference. Nutrients 12 (10), 3197. doi:10.3390/nu12103197

Watson, A. J., and Duckworth, C. A. (2010). Gut Microbiota Control Gut Permeability through GLP-2. Gastroenterology 138 (2), 779-781. doi:10.1053/j.gastro.2009.12.017

Wei, Y., Yang, H., Zhu, C., Deng, J., and Fan, D. (2020). Hypoglycemic Effect of Ginsenoside Rg5 Mediated Partly by Modulating Gut Microbiota Dysbiosis in Diabetic Db/db Mice. J. Agric. Food Chem. 68 (18), 5107-5117. doi:10.1021/ acs.jafc.0c00605

Wu, G. (2013). Functional Amino Acids in Nutrition and Health. Amino Acids 45 (3), 407-411. doi:10.1007/s00726-013-1500-6

Xiao, N., Lou, M. D., Lu, Y. T., Yang, L. L., Liu, Q., Liu, B., et al. (2017). Ginsenoside Rg5 Attenuates Hepatic Glucagon Response via Suppression of SuccinateAssociated HIF-1a Induction in HFD-Fed Mice. Diabetologia 60 (6), 1084-1093. doi:10.1007/s00125-017-4238-y

Xiao, S., Zhang, Z., Chen, M., Zou, J., Jiang, S., Qian, D., et al. (2019). Xiexin Tang Ameliorates Dyslipidemia in High-Fat Diet-Induced Obese Rats via Elevating Gut Microbiota-Derived Short Chain Fatty Acids Production and Adjusting Energy Metabolism. J. Ethnopharmacol. 241, 112032. doi:10.1016/ j.jep.2019.112032

Xiong, X. Q., Geng, Z., Zhou, B., Zhang, F., Han, Y., Zhou, Y. B., et al. (2018). FNDC5 Attenuates Adipose Tissue Inflammation and Insulin Resistance via 
AMPK-Mediated Macrophage Polarization in Obesity. Metabolism 83, 31-41. doi:10.1016/j.metabol.2018.01.013

Xu, J., Li, T., Xia, X., Fu, C., Wang, X., and Zhao, Y. (2020a). Dietary Ginsenoside T19 Supplementation Regulates Glucose and Lipid Metabolism via AMPK and PI3K Pathways and its Effect on Intestinal Microbiota. J. Agric. Food Chem. 68 (49), 14452-14462. doi:10.1021/acs.jafc.0c04429

Xu, Y., Wang, N., Tan, H. Y., Li, S., Zhang, C., and Feng, Y. (2021). Gut-liver axis Modulation of Panax Notoginseng Saponins in Nonalcoholic Fatty Liver Disease. Hepatol. Int. 15 (2), 350-365. doi:10.1007/s12072-021-10138-1

Xu, Y., Wang, N., Tan, H. Y., Li, S., Zhang, C., Zhang, Z., et al. (2020b). Panax Notoginseng Saponins Modulate the Gut Microbiota to Promote Thermogenesis and Beige Adipocyte Reconstruction via Leptin-Mediated AMPKa/STAT3 Signaling in Diet-Induced Obesity. Theranostics 10 (24), 11302-11323. doi:10.7150/thno.47746

Yang, L., Zou, H., Gao, Y., Luo, J., Xie, X., Meng, W., et al. (2020). Insights into Gastrointestinal Microbiota-Generated Ginsenoside Metabolites and Their Bioactivities. Drug Metab. Rev. 52 (1), 125-138. doi:10.1080/03602532.2020.1714645

Yang, Q., Vijayakumar, A., and Kahn, B. B. (2018). Metabolites as Regulators of Insulin Sensitivity and Metabolism. Nat. Rev. Mol. Cel Biol. 19 (10), 654-672. doi:10.1038/s41580-018-0044-8

Yao, F., Xue, Q., Li, K., Cao, X., Sun, L., and Liu, Y. (2019). Phenolic Compounds and Ginsenosides in Ginseng Shoots and Their Antioxidant and Antiinflammatory Capacities in Lps-Induced raw264.7 Mouse Macrophages. Int. J. Mol. Sci. 20 (12), 2951. doi:10.3390/ijms20122951

Yoneshiro, T., Wang, Q., Tajima, K., Matsushita, M., Maki, H., Igarashi, K., et al. (2019). BCAA Catabolism in Brown Fat Controls Energy Homeostasis through SLC25A44. Nature 572 (7771), 614-619. doi:10.1038/s41586-019-1503-x

Yue, S. J., Liu, J., Wang, A. T., Meng, X. T., Yang, Z. R., Peng, C., et al. (2019). Berberine Alleviates Insulin Resistance by Reducing Peripheral BranchedChain Amino Acids. Am. J. Physiol. Endocrinol. Metab. 316 (1), E73-E85. doi:10.1152/ajpendo.00256.2018

Zeng, S. L., Li, S. Z., Xiao, P. T., Cai, Y. Y., Chu, C., Chen, B. Z., et al. (2020). Citrus Polymethoxyflavones Attenuate Metabolic Syndrome by Regulating Gut Microbiome and Amino Acid Metabolism. Sci. Adv. 6 (1), eaax6208. doi:10.1126/sciadv.aax6208

Zhang, F., Tang, S., Zhao, L., Yang, X., Yao, Y., Hou, Z., et al. (2021). Stem-leaves of Panax as a Rich and Sustainable Source of Less-Polar Ginsenosides:
Comparison of Ginsenosides from Panax Ginseng, American Ginseng and Panax Notoginseng Prepared by Heating and Acid Treatment. J. Ginseng Res. 45 (1), 163-175. doi:10.1016/j.jgr.2020.01.003

Zhang, H., Qi, R., Zeng, Y., Tsao, R., and Mine, Y. (2020a). Chinese Sweet Leaf tea (Rubus Suavissimus) Mitigates LPS-Induced Low-Grade Chronic Inflammation and Reduces the Risk of Metabolic Disorders in a C57BL/6J Mouse Model. J. Agric. Food Chem. 68 (1), 138-146. doi:10.1021/ acs.jafc.9b05975

Zhang, L., Yue, Y., Shi, M., Tian, M., Ji, J., Liao, X., et al. (2020b). Dietary Luffa Cylindrica (L.) Roem Promotes Branched-Chain Amino Acid Catabolism in the Circulation System via Gut Microbiota in DietInduced Obese Mice. Food Chem. 320, 126648. doi:10.1016/ j.foodchem.2020.126648

Zhou, S. S., Auyeung, K. K., Yip, K. M., Ye, R., Zhao, Z. Z., Mao, Q., et al. (2020). Stronger Anti-obesity Effect of white Ginseng over Red Ginseng and the Potential Mechanisms Involving Chemically Structural/compositional Specificity to Gut Microbiota. Phytomedicine 74, 152761. doi:10.1016/ j.phymed.2018.11.021

Conflict of Interest: The authors declare that the research was conducted in the absence of any commercial or financial relationships that could be construed as a potential conflict of interest.

Publisher's Note: All claims expressed in this article are solely those of the authors and do not necessarily represent those of their affiliated organizations, or those of the publisher, the editors and the reviewers. Any product that may be evaluated in this article, or claim that may be made by its manufacturer, is not guaranteed or endorsed by the publisher.

Copyright $\odot 2021$ Zhuang, Li, Yang, Wang, Ding and Zhou. This is an open-access article distributed under the terms of the Creative Commons Attribution License (CC $B Y)$. The use, distribution or reproduction in other forums is permitted, provided the original author(s) and the copyright owner(s) are credited and that the original publication in this journal is cited, in accordance with accepted academic practice. No use, distribution or reproduction is permitted which does not comply with these terms. 